\title{
Primary Hyperparathyroidism in Pregnancy: Insights From a Case of a 28-Year-Old Woman With Miscarriages and Hyperemesis Gravidarum
}

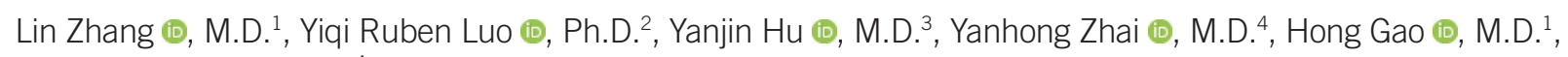
and Zheng Cao $\mathbb{E}$, Ph.D. ${ }^{4}$

${ }^{1}$ Department of Internal Medicine, Beijing Obstetrics and Gynecology Hospital, Capital Medical University, Beijing, China; ${ }^{2}$ Department of Laboratory Medicine, University of California San Francisco, San Francisco, CA, USA; ${ }^{3}$ Department of Endocrinology, Beijing Chao-yang Hospital, Capital Medical University, Beijing, China; ${ }^{4}$ Department of Laboratory Medicine, Beijing Obstetrics and Gynecology Hospital, Capital Medical University, Beijing, China

\section{Dear Editor,}

Serum calcium level usually decreases during pregnancy due to hemodilution; thus, hypercalcemia in pregnancy should be followed by parathyroid hormone (PTH) measurement to exclude parathyroid dysfunction. We report a case of severe hyperemesis gravidarum combined with hypercalcemia that led to two consecutive pregnancy losses. This report was approved by the Ethics Committee of Beijing Obstetrics and Gynecology Hospital, Beijing, China (2017-KY-011-02) and was conducted in accordance with the 1964 Helsinki Declaration and its amendments. The study protocol was approved by the Institutional Committee on human research. The patient provided written informed consent to publish her case. In July 2019, a 28-year-old pregnant woman at 13 gestational weeks (GWs) presented to the Internal Medicine Department of Beijing Obstetrics and Gynecology Hospital, Beijing, China, with a chief complaint of intermittent nausea and vomiting. During this visit, the patient tested positive for urine ketone bodies $(+4)$, hypokalemia, and hypercalcemia (Table 1). Neither kidney stone nor nephrocalcinosis was found in abdominal ultrasound examinations. After necessary nutritional supplementation, she tested negative for urinary ketone bodies. With a diagnosis of hyperemesis gravidarum and hypercalcemia of unknown origin, the patient was discharged and referred to the Endocrinology Department for further evaluation. Notably, the patient was diagnosed as having hyperemesis gravidarum accompanied by hypercalcemia, and she had a miscarriage at $10 \mathrm{GWs}$ three years ago.

The patient consulted an endocrinologist at GW 14, and her laboratory workup results showed elevated intact PTH (iPTH) and serum calcium levels, and low serum phosphorus level (Table 1). Ultrasonography of the parathyroid gland revealed a lesion of approximately $0.6 \times 0.4 \mathrm{~cm}$ at the posterior pole of the left lobe of the parathyroid. The lesion was hypoechoic and inhomogeneous, with a clear border and visible blood flow signals
Received: July 14, 2020

Revision received: August 2, 2020

Accepted: November 11, 2020

Corresponding author: Zheng Cao, Ph.D.

Department of Laboratory Medicine, Beijing Obstetrics and Gynecology Hospital, Capital Medical University, 251 Yaojiayuan Road, Beijing 100026, China

Tel: +86-10-52276406, Fax: +86-10-52276413

E-mail: zhengcao2011@hotmail.com

Co-corresponding author: Hong Gao, M.D.

Department of Internal Medicine, Beijing Obstetrics and Gynecology Hospital, Capital Medical University, 251 Yaojiayuan Road, Beijing 100026, China

Tel: +86-10-52276406, Fax: +86-10-52276502, E-mail: helen31198@163.com

\section{(i) $\$$}

\section{(C) Korean Society for Laboratory Medicine}

This is an Open Access article distributed under the terms of the Creative Commons Attribution Non-Commercial License (https://creativecommons.org/licenses/by-nc/4.0) which permits unrestricted non-commercial use, distribution, and reproduction in any medium, provided the original work is properly cited. 
detected by color Doppler flow imaging (HITACHI Ascendus, Tokyo, Japan). These results were consistent with a diagnosis of parathyroid hyperplasia or primary hyperparathyroidism (PHPT) in light of the hypercalcemia and increased serum iPTH level. At GW 17, ultrasound-guided microwave ablation (MWA) of the parathyroid gland was performed under local anesthesia with lidocaine. Although the patient's iPTH, serum calcium, and phosphorus levels returned to normal shortly after the intervention (Table 1), she was admitted again at GW 19 for severe malnutrition and experienced miscarriage followed by induced abortion.

PHPT causes excessive PTH secretion, which can lead to osteoporosis, kidney stones, fragile fracture, high calcium crisis, and serious maternal and fetal adverse outcomes during pregnancy [1]. A previous study indicated that $68 \%$ of pregnant women with PHPT experienced maternal complications such as preeclampsia, polyhydramnios, and spontaneous abortion, and $80 \%$ suffered fetal/neonatal complications such as fetal growth restriction, intrauterine fetal death, neonatal tetany, and neonatal death [2]. Among cases of miscarriage associated with PHPT, the severity of the complications was positively correlated with the elevated serum calcium levels [3]. More strikingly, $72 \%$ of pregnant women with PHPT have had at least one spontaneous miscarriage [4]. However, the common but non-specific clinical

Table 1. Serum calcium, phosphorus, and iPTH levels in the clinical course of the patient

\begin{tabular}{|c|c|c|c|c|c|}
\hline \multirow{2}{*}{$\frac{\text { Major clinical event }}{\text { Gestational weeks }}$} & \multicolumn{2}{|c|}{ Pre-MWA } & \multicolumn{2}{|c|}{ Post-MWA } & \multirow{2}{*}{$\frac{\text { Miscarriage }}{21}$} \\
\hline & 13 & 14 & 17 & 19 & \\
\hline Serum calcium $(\mathrm{mmol} / \mathrm{L})^{*}$ & 2.87 & 2.72 & 1.78 & 1.82 & 1.81 \\
\hline Serum phosphorus (mmol/L)* & 0.58 & 0.62 & 0.56 & 0.88 & 1.14 \\
\hline iPTH (pg/mL)* & & 165.3 & 27.3 & & \\
\hline
\end{tabular}

${ }^{*}$ Reference intervals: serum calcium, 2.10-2.55 mmol/L; serum phosphorus, 0.74-1.52 mmol/L; iPTH, 12-88 pg/mL.

Abbreviations: iPTH, intact parathyroid hormone; MWA, microwave ablation. presentations of PHPT, such as nausea, vomiting, and fatigue, are similar to those of pregnancy, and therefore PHPT diagnosis is often missed or inappropriately treated as plain hyperemesis gravidarum. An additional challenge in the diagnosis of PHPT in pregnancy is that serum calcium is physiologically hemodiluted during pregnancy, and thus its pathological increase is much less noticeable.

Surgery is recommended for patients with PHPT in pregnancy, if the serum calcium level is $\geq 2.85 \mathrm{mmol} / \mathrm{L}$; if it is $<2.85 \mathrm{mmol} / \mathrm{L}$, conservative treatment such as a low calcium diet, hydration, diuresis, and/or calcitonin administration should be considered [5]. Some experts have suggested that surgery should be performed when the serum calcium level is $>3 \mathrm{mmol} / \mathrm{L}$, and that the intervention strategy should be determined by the gestational stage, hypercalcemia severity and symptoms, and patient preference [6]. In the present case, the patient received MWA instead of conventional surgery to avoid potential adverse effects due to general anesthesia, surgery wound, and bleeding. MWA has been successfully adopted as a minimally invasive therapeutic alternative to surgery for some patients, including pregnant women, who are intolerant to or unwilling to undergo parathyroidectomy under general anesthesia [7].

A literature review revealed three similar reports on hyperemesis gravidarum combined with PHPT in pregnancy (Table 2) [8 10]. All three cases underwent parathyroidectomy but had diverse pregnancy outcomes. In the present case (case 4 in Table 2), miscarriage occurred three weeks after MWA, which was likely due to the long-term malnutrition and hyperparathyroidism condition.

In summary, serum calcium and PTH levels should be monitored in pregnant women who present with severe and constant hyperemesis gravidarum to exclude the possibility of PHPT, which poses a significant risk of maternal/fetal complications, including miscarriage.

Table 2. Clinical cases of PHPT with hyperemesis gravidarum in pregnancy

\begin{tabular}{lcccc}
\hline Characteristics & Case 1 [8] & Case 2 [9] & Case 3 [10] & Case 4 [Present case] \\
\hline Age (yr) & 27 & 22 & 23 & G1P0 \\
Gestation history & G1P0 & G2P1 & 3.19 & G2P0 \\
Average serum calcium level (mmol/L) & 2.92 & 4.38 & 11 & 2.96 \\
Gestational age of diagnosis (week) & 17 & Parathyroidectomy & Parathyroidectomy & Uncomplicated \\
Intervention & Parathyroidectomy & Preeclampsia & Intrauterine death \\
Pregnancy outcome & Intrauterine death & Cesarean section & Vaginal delivery & Miscarriage \\
Delivery outcome & Miscarriage & grave
\end{tabular}

Abbreviations: PHPT, primary hyperparathyroidism; G, gravidity; P, parity; MWA, microwave ablation. 


\section{ACKNOWLEDGEMENTS}

None.

\section{AUTHOR CONTRIBUTIONS}

Study conception and design: Zhang L, Gao H, and Cao Z. Data acquisition and analysis: Zhang $\mathrm{L}$ and $\mathrm{Hu} Y$. Analysis and interpretation of the data: Zhang L, Zhai Y, and Cao Z. Writing the manuscript: Zhang $L$ and Cao $Z$. Reviewing and editing the manuscript: Zhang L, Luo YR, Zhai Y, and Cao Z. Final approval of the manuscript: Zhang L, Hu Y, Luo YR, Zhai Y, Gao H, and Cao Z.

\section{CONFLICTS OF INTEREST}

The authors state that there are no conflicts of interest with regard to publication of this article. The funding organizations played no role in the study design; in the collection, analysis, and interpretation of data; in the writing of the report; or in the decision to submit the report for publication.

\section{RESEARCH FUNDING}

This work was supported by the Beijing Obstetrics and Gynecology Hospital, Capital Medical University (No. FCYY201624) and the National Natural Science Foundation of China (No. 81702057).

\section{ORCID}

Lin Zhang

https://orcid.org/0000-0001-5386-3983

Yiqi Ruben Luo
Yanjin $\mathrm{Hu}$

https://orcid.org/0000-0002-3124-7132

Yanhong Zhai

Hong Gao

Zheng Cao

https://orcid.org/0000-0002-7575-7396

https://orcid.org/0000-0002-0421-7579

https://orcid.org/0000-0002-0968-1864

\section{REFERENCES}

1. Song A, Wang W, Chen S, Wang Y, Liu S, Nie M, et al. Primary hyperparathyroidism during pregnancy: a case series of 8 patients. Endocr Pract 2019;25:1127-36.

2. Schnatz PF and Thaxton S. Parathyroidectomy in the third trimester of pregnancy. Obstet Gynecol Surv 2005;60:672-82.

3. Malekar-Raikar S and Sinnott BP. Primary hyperparathyroidism in pregnancy-a rare cause of life-threatening hypercalcemia: case report and literature review. Case Rep Endocrinol 2011;2011:520516.

4. DiMarco AN, Meeran K, Christakis I, Sodhi V, Nelson-Piercy C, Tolley NS, et al. Seventeen cases of primary hyperparathyroidism in pregnancy: a call for management guidelines. J Endocr Soc 2019;3:1009-21.

5. Norman J, Politz D, Politz L. Hyperparathyroidism during pregnancy and the effect of rising calcium on pregnancy loss: a call for earlier intervention. Clin Endocrinol (Oxf) 2009;71:104-9.

6. Khan AA, Hanley DA, Rizzoli R, Bollerslev J, Young JE, Rejnmark L, et al. Primary hyperparathyroidism: review and recommendations on evaluation, diagnosis, and management. A Canadian and international consensus. Osteoporos Int 2017;28:1-19.

7. Wei Y, Peng L, Li Y, Zhao ZL, Yu MA. Clinical study on safety and efficacy of microwave ablation for primary hyperparathyroidism. Korean J Radio 2020;21:572-81.

8. Stahl J, Winters N, Shariff A. A rare case of primary hyperparathyroidism, hyperemesis gravidarum, and Wernicke encephalopathy. AACE Clin Case Rep 2019;5:e108-11.

9. Yilmaz BA, Altay M, Değertekin CK, Çimen AR, lyidir OT, Biri A, et al. Hyperparathyroid crisis presenting with hyperemesis gravidarum. Arch Gynecol Obstet 2014;290:811-4.

10. Benson BC, Guinto RE, Parks JR. Primary hyperparathyroidism mimicking hyperemesis gravidarum. Hawaii J Med Public Health 2013;72: 11-3. 